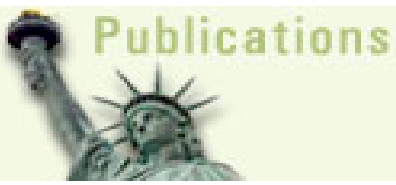

American Journal of Biochemistry and Biotechnology 3 (4): 193-198, 2007

ISSN 1553-3468

(C) 2007 Science Publications

\title{
Identification and functional characterization of a novel lysine-rich protein from Entamoeba histolytica
}

\author{
${ }^{1}$ Alka Mehra, ${ }^{2}$ Sudha Bhattacharya and ${ }^{1,3}$ Alok Bhattacharya \\ ${ }^{1}$ School of Life Sciences, ${ }^{2}$ School of Environmental Sciences and ${ }^{3}$ School of Information \\ Technology, Jawaharlal Nehru University, New Delhi, India
}

\begin{abstract}
In an attempt to understand the role of genes with no known function, in the protozoan parasite Entamoeba histolytica, an analysis of the proteome was carried out and a Lysine-Rich protein (KRP) was identified for further study. This protein has a modular structure with four domains and was found to be expressed and localized to the cell surface of E. histolytica trophozoites. The KRP over expressing cells were $80 \%$ less virulent as compared to the cells under expressing the gene as determined by the ability of the cells to kill in vitro target cells. The results suggest that KRP may have a role in the cytopathogenesis of the parasite.
\end{abstract}

Key words: Entamoeba histolytica, lysine, serine, surface protein, lysozyme, cytopathic

\section{INTRODUCTION}

The cell surface of the protozoan parasite Entamoeba histolytica has been characterized using a number of approaches and attempts have been made to associate pathogenicity with presence or absence of a few molecules or with some cell surface characteristics $^{[1,2,3]}$. Among many reported cell surface proteins only a few have been characterized in detail ${ }^{[4]}$. Some of the molecules, such as SREHP (serine-rich $E$. histolytica protein), Ariel (asparagine rich antigen) are immunogenic and being used either as diagnostic marker for invasive disease or have potential to be a vaccine candidate ${ }^{[5,6.7]}$. The SREHP and Ariel proteins have multiple tandem repeats consisting of hydrophilic octapeptide sequences E-A-S-S-S/T-D/N-K-P and D/N/S-E-S-S-D/N-N-K-P respectively ${ }^{[8,9,10]}$. They also possess $\mathrm{N}$-terminal signal and C-terminal hydrophobic anchor sequences. The structural motifs of SREHP resemble some of the repetitive antigens of malarial parasite, especially the circumsporozoite protein ${ }^{[11]}$. SREHP is known to show extensive polymorphism particularly in the repetitive region. These polymorphisms have been used to type clinical isolates and attempts have been made to link the polymorphisms with clinical features of the patients $^{[6,12]}$. Homolog of SREHP is also found in nonpathogenic species E. dispar. The Ariel protein family is polymorphic with varying number of octapeptide repeats in different members ${ }^{[10]}$. This gene family was reported to be absent in the nonpathogenic species Entamoeba dispar ${ }^{[3]}$. In this report we describe one such protein, the lysine rich protein (KRP), containing a serine-rich repetitive motifs and a lysinerich domain and show that it may be functionally important for E. histolytica.

\section{MATERIALS AND METHODS}

Nucleotide and protein sequences of all target genes were retrieved from the E. histolytica genome database (http://www.ncbi.nlm.nih.gov). The BLAST sequence similarity search was done against the $E$. histolytica genome database (12.5X coverage, that is the final assembled genome sequence) at The Institute of Genome Research (http://www.tigr.org/tdb/e2k1/eha1), the Wellcome Trust's Sanger (http://www.sanger.ac.uk/Projects/E histolytica) and NCBI. Determination of amino acid composition was performed using a set of tools available at www.expasy.ch.

All experiments were carried out with $E$. histolytica strain HM-1:IMSS clone 6 as described before ${ }^{[13]}$. Cell viability was determined by microscopy using trypan blue dye exclusion test. CV1 (Monkey African Green kidney fibroblast) cell line was cultured in Dulbecco's modified Eagle's medium (DMEM) (Gibco) supplemented with 9\% fetal bovine serum, penicillin and streptomycin. Transfection of $E$. histolytica trophozoites was performed by electroporation as described previously ${ }^{13]}$.

Northern Analysis: RNA was isolated from $E$. histolytica strain HM1: IMSS using Tripure reagent (Roche, USA) as per the manufacturer's protocol. Thirty microgram of total RNA from mid log phase cells was resolved on a $1.2 \%$ denaturing agaroseformaldehyde gel and blotted onto a nylon membrane.

Corresponding Author: Alok Bhattacharya, School of Life Sciences, Jawaharlal Nehru University, New Delhi, India. Tel.: +91 112670 4516; Fax: +911126717586 
The blot was hybridized with a full length KRP radiolabeled probe in a hybridization buffer containing $1 \% \mathrm{SDS}$ and $1 \mathrm{M} \mathrm{NaCl}$ at $65^{\circ} \mathrm{C}$ for $16 \mathrm{~h}$. Radiolabeling of the DNA was carried out in presence of Klenow fragment of DNA polymerase using random primers (NEB labeling kit). The hybridization signal was seen by autoradiography and was observed to be $2.2 \mathrm{~kb}$ in size.

Western Analysis: The total cell lysate of $E$. histolytica trophozoites was prepared by solubilizing the cell pellet in a lysis buffer containing $10 \mathrm{mM}$ Tris$\mathrm{Cl}$, pH 7.5, 150mM NaCl, 1\% Triton X-100 (w/v) with protease inhibitors like $2 \mathrm{mM}$ PMSF, $10 \mu \mathrm{g} / \mathrm{ml}$ leupeptin, $10 \mu \mathrm{g} / \mathrm{ml}$ aprotonin, $1 \mathrm{mM}$ iodoacetamide, 5mM PHMB, 2mM EDTA, $0.1 \mathrm{mM}$ E-64) followed by centrifugation at $10,000 \mathrm{xg}, 2 \mathrm{~min}, 4^{\circ} \mathrm{C}$ and the supernatant was stored frozen in aliquots. Hundred micrograms of the total cell lysate was resolved on a 10 $\%$ SDS-PAGE gel and transferred onto a nitrocellulose membrane. The blot was incubated with indicated sera followed by secondary anti-rabbit HRPO and was developed using ECL chemiluminescence kit.

Immunostaining: E. histolytica trophozoites were fixed with $3.7 \%$ paraformaldehyde. The fixed cells were permeablized with $0.1 \%$ TritonX-100/PBS as indicated and were blocked in 3\% BSA/PBS followed by immunostaining with indicated antibody. The bound antibodies were visualized by staining with Alexa 488labeled-goat anti rabbit secondary antibody. The images were captured at 100x magnification (Zeiss LSM Meta model).

Cell Fractionation: The total cell lysate of $E$. histolytica trophozoites was prepared by suspending the cell pellet in a lysis buffer containing $10 \mathrm{mM}$ Tris-Cl, $\mathrm{pH} 7.5,150 \mathrm{mM} \mathrm{NaCl}$, containing protease inhibitors like $2 \mu \mathrm{mM}$ PMSF, $10 \mu \mathrm{g} / \mathrm{ml}$ leupeptin, $10 \mu \mathrm{g} / \mathrm{ml}$ aprotonin, $1 \mathrm{mM}$ iodoacetamide, $5 \mathrm{mM}$ PHMB, $2 \mathrm{mM}$ EDTA, $0.1 \mathrm{mM}$ E-64 followed by freeze thaw thrice. This was then sonicated on ice for $10 \mathrm{sec}$ with 1 min gap twice. The cell lysate was thereafter fractionated by ultracentrifugation at $1,00,000 \mathrm{xg}$ into membranes and cytosol.

\section{RESULTS AND DISCUSSION}

The lysine and serine constitute $17.9 \%$ and $11.3 \%$ of the lysine-rich protein (KRP) respectively. It is annotated as a putative lysozyme in the Entamoeba genome database with an accession number XM 649403. Computational analysis involving a number of tools predicted a modular organization for KRP with four distinct regions (Fig 1A). The amino acid residues 24 to 196 displayed $41 \%$ identity $\left(\mathrm{E}=2 \mathrm{X} 10^{-31}\right)$ with $\mathrm{N}$-Acetyl muraminidase (Accession No. CAA60916) of E. histolytica. The Entamoeba genome harbours two genes that are thought to have lysozyme-like activity (CAA60916 and AAC672351).
However these are likely to be unconventional as both the biochemical properties and aminoacid sequence do not resemble the lysozymes of other organisms ${ }^{[14,15]}$. Apart from KRP, five other genes have been annotated as putative lysozymes. The predicted lysozyme domain of KRP, annotated as lysozyme M1 (1,4-beta-Nacetylmuramidase) is different from known amebic lysozymes in terms of degree of sequence identity $(41 \%)$ and the presence of additional domains. Analysis of the active site residues indicated a glutamate and an aspartate as active site residues of lysozyme ${ }^{[16]}$. The lysozyme domain of KRP has the conserved aspartate but not glutamate. Moreover, motifs generated from multiple alignment of amebic and other protozoan lysozyme-like proteins identified amebic lysozyme-like proteins and a few similar proteins from a few protozoa (data not shown here). Instead the search picked up a few of glycosyl hydrolases from a few protozoa. Therefore, we conclude that it is highly unlikely that this domain is still functional as lysozyme. The fused domain has accumulated changes considerably as the fusion event may have occurred quite some time back. At present the activity of the recombinant KRP has not been checked in order to confirm our prediction. The other domains present are the hydrophobic N-terminal signal sequence (1-24 amino acid residues), the central serine rich region (224 to 366 amino acid residues) and positively charged region (367-748 amino acid residues). The charged region contains abundant lysines and arginines. The lysine rich domain contributes to overall basic nature of this protein. The central serinerich domain has a number of different types of novel tandem repeats (Fig. 1B). The sequence analysis did not reveal any hydrophobic sequence either internally or at the C-terminal, which could serve as a cell surface anchor. It is possible that KRP may undergo lipid modification that can help it to get anchored on to the plasma membrane.

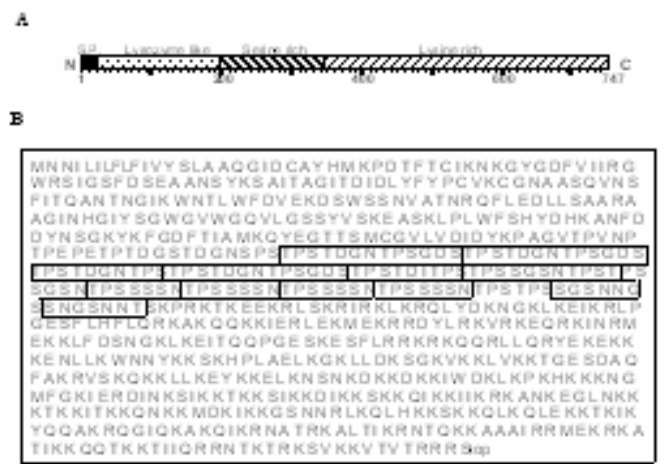

Fig. 1: Sequence organization, expression and localization of KRP

A. Schematic representation of the different domains of KRP, which have been drawn to 
scale. The numbers below the line indicate the amino acid residues. S.P. is the signal peptide.

B. Amino acid sequence of KRP. The tandem imperfect repeats in the serine-rich domain are enclosed in boxes. There are six tandem imperfect repeats with the repeat motif of STPSTDGNTPSGD. These are followed by four perfect repeats of NTPSSSS. These repeats are followed by two imperfect repeats.

Sequence analysis suggested that there is no homolog of KRP in other organisms, in terms of sequence similarity and domain organization. Interspecies conservation of genes can often be indicative of functional importance. In order to find out if an ortholog of this protein exists in any other species of Entamoeba, sequence similarity searches were carried out. A putative homolog showing similarity across the major part of the protein was found in non pathogenic sibling species Entamoeba dispar (contig aanv01000649). However, the first exon and intron were not found. This region encodes the signal sequence and part of the lysozyme domain. Since the $E$. dispar protein may be cytoplasmic without the intact lysozyme domain it is likely this domain may have a role in pathogenesis.

The presence of an ORF alone is not sufficient to indicate that E. histolytica possesses a functional KRP protein. Often computational prediction of genes is inaccurate. In order to show that the sequence is not a pseudogene, the expression of this gene was checked in actively growing trophozoites by northern and western analysis. Northern analysis revealed an RNA band of $2.2 \mathrm{~kb}$ (Fig. 1C). The band was of expected size based on the transcription of the entire ORF. Western blot analysis was carried out using an antibody raised against the recombinant serine-rich domain (amino acid residue 188 to 349 ). A $85 \mathrm{kDa}$ band was observed. This was close to the theoretical size of $82 \mathrm{kDa}$ (Fig. 1D). Thus, the full length KRP is expressed in the trophozoites of E. histolytica, suggesting that the theoretical prediction is accurate and the gene is functionally active. This is important as the assembly of the E. histolytica genome may still not be final and there may be errors due to highly repetitive nature of the genome. The antibody (polyclonal anti-KRP raised in rabbit) was also used to localize KRP protein in the cell. Western blot analysis using fractionated cell lysate showed immunoreactivity to be present in the membrane fraction (Fig. 1E). This was further confirmed by immunofluorescence. The staining was found to be associated with the plasma membrane suggesting it to be a cell surface protein (Fig. 1F).
A

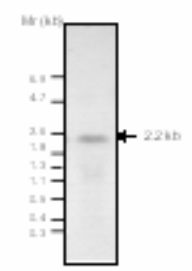

c

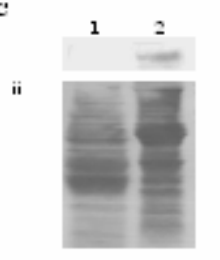

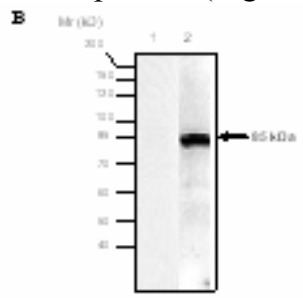

D

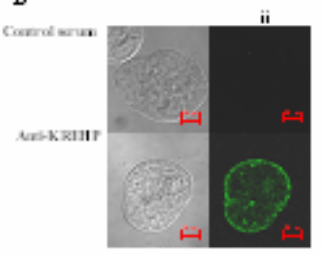

Fig. 2:

A. Expression of KRP in E. histolytica trophozoites by Northern Analysis. The hybridization signal was seen by autoradiography and was observed to be 2.2 $\mathrm{kb}$ in size.

B. Expression of KRP in E. histolytica trophozoites by Western Analysis. The antiKRP was raised by immunizing rabbit with the purified recombinant central serine rich domain. The lane 1: control serum and lane 2: anti-KRP antibody.

C. Subcellular localization of KRP by Western Analysis and cell fractionation. Hundred micrograms of the different fraction was analyzed by western blotting with anti-KRP antibody (Panel i). The parallel lanes were stained with Coomassie blue to indicate the amount of protein loaded onto each lane (Panel ii). Lane 1: cytosol and Lane 2: membranes. 
D. Immunostaining. The panel (i) shows the phase contrast images while panel (ii) shows the fluorescent images. Bar: $10 \mu \mathrm{m}$.

The data presented above indicated that KRP may be one of the genes that do not have any known function. Therefore, to understand the function of this gene a functional genomic approach was used. Since the ploidy of E. histolytica is high and no method is yet available for integrating DNA fragments to chromosomes, an approach was taken to overexpress and underexpress the gene in E. histolytica cells using a constitutive expression vector [13]. The gene was cloned in both sense (pKRP-S) and antisense (pKRPAS) orientation (see Fig. 2A) using a PCR based approach as described in the figure legend. The orientation of the constructs was confirmed by sequencing. These constructs were transfected into $E$. histolytica cells to establish EhKRP-S and EhKRP-AS cell lines which were maintained in the presence of 10 $\mu \mathrm{g} / \mathrm{ml} \mathrm{G} 418$. All the experiments were carried out with transfectants grown in the presence of $50 \mu \mathrm{g} / \mathrm{ml} \mathrm{G} 418$ for $48 \mathrm{~h}$. The level of the KRP transcript in the transformed cell lines was checked by northern analysis using single stranded probes (Fig. 2B). The antisense probe hybridized to a transcript of about 550 nucleotides derived from EhKRP-AS cells whereas no antisense transcript was observed in EhKRP-S cells (Fig. 2B, i). On the other hand sense strand-specific probe did not hybridize to any band in the antisense cell line but hybridized to a $2.2 \mathrm{~kb}$ band, corresponding to the full length transcript, from an RNA preparation derived from EhKRP-S cells (Fig. 2B, ii). These results confirmed that the transformants expressed the cloned KRP gene in the expected manner. This was further confirmed by western analysis of the total cell lysates from EhKRP-S and EhKRP-AS cell lines using antiKRP antibody (Fig. 2C).

Different parameters such as growth, erythrophagocytosis and cytopathic ability were used to assess phenotypic changes in the EhKRP-S and EhKRP-AS cells. These properties are often used as markers for assessing the virulent potential of $E$. histolytica cells $[17,18,19]$. The results of a cytopathic assay using the CV1 monolayer is shown in Fig. 2D. The data is represented as percentage of monolayer destruction as compared to the untransfected cells. The E. histolytica cells transfected with the parental plasmid, pEh-Neo-GFP (Ehgfp) and grown in the presence of $50 \mu \mathrm{g} / \mathrm{ml}$ of $\mathrm{G} 418$ for $48 \mathrm{~h}$ were also used as a control in the experiment. Interestingly, overexpression of KRP led to $80 \%$ decrease in the cytopathic property. On the other hand, there was only $20-30 \%$ reduction in the ability of EhKRP-AS cells to kill CV-1 cells. No significant difference was observed in erythrophagocytosis and cell proliferation among the three cell lines (data not shown). This data suggests that KRP may be involved in modulation of cytopathic properties of E. histolytica. It appears that it may not be directly involved in cytopathogenesis, as there was only $20-30 \%$ reduction in cell killing on antisense blocking by antisense approach. Absence of this gene in nonpathogenic species of Entamoeba also supports the suggestion that this gene has a role in cytopathogenesis.

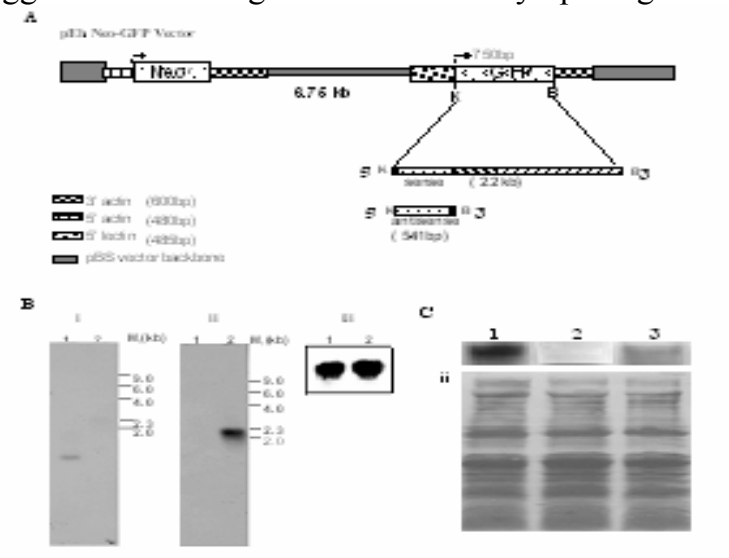

Fig. 3: Overexpression and underexpression of KRP in E. histolytica trophozoites and cytopathic assay.

A. Schematic representation of the constitutive expression vector $\mathrm{pEh}$ Neo-GFP used for transfection. The GFP gene of the shuttle vector $\mathrm{pEh}$ Neo-GFP was excised by the KpnI $(\mathrm{K})$ and BamHI (B) and replaced with the $k r p$ gene in the sense orientation or antisense orientation. Appropriate restriction sites were employed in the primers used for PCR amplification of the krp gene for cloning in the sense and the antisense orientation. Only initial $541 \mathrm{bp}$ of the krp gene was amplified and used for antisense blocking of the KRP expression. These constructs were stably transfected in E. histolytica trophozoites and all the experiments were carried out with cell lines grown in presence of $50 \mu \mathrm{g} / \mathrm{ml}$ of G418 for $48 \mathrm{~h}$.

B. Northern analysis of the cell lines transfected with EhKRP-S and EhKRP-AS construct. Lane1: RNA from Eh KRP-AS, lane 2: RNA 
from Eh KRP-S. Hybridization with rDNA probe was used to show equal loading of the RNA. The probes used are as indicated:

$i$, antisense strand-specific; ii, sense strandspecific; iii, rRNA.

C. Western analysis of the cell lines transfected with EhKRP-S and EhKRP-AS construct. The membrane was incubated with anti KRP antibody and further stained with the HRPOlabeled second antibody (Panel i). The parallel lanes were stained with Coomassie blue to check for equal loading (Panel ii). Lane 1: EhKRP-S, lane 2: EhKRP-AS, lane 3: Ehgfp transfectants.

There is not enough data to speculate about the mechanism by which KRP may influence virulence properties of E. histolytica. It is likely that KRP inhibits target cell killing by mopping certain virulence effector molecules. Therefore, overexpression of this molecule induces the dominant negative phenotype. At low level of expression in normal cells it may have other functions. Three of the genes that contain regions rich in charged amino acids, such as lysine and glutamic acid are down regulated in abscess-derived trophozoites of E. histolytica ${ }^{[20]}$. Other than shared repetitive stretches of the amino acid sequence PKKEEKKP, there was no similarity among these proteins suggesting that proteins containing charged amino acid-rich domains may play an important role in virulence ${ }^{[20]}$. The other likely mechanism can be due to its ability to block cell surface signaling if present in high numbers. Detailed experiments are underway to understand this phenomenon. Further studies on the function of this protein may throw important light on its role in pathogenesis.

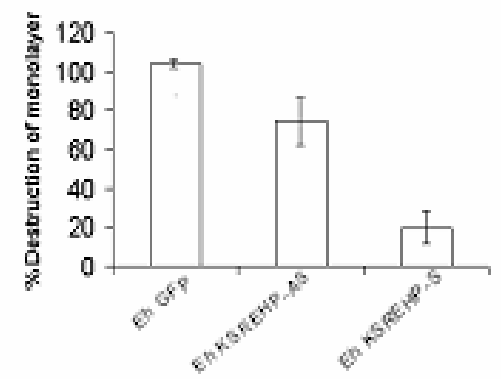

Fig. 4: Cytopathic properties of EhKRP-AS and EhKRP-S cells. The indicated transfectants EhKRP-S, EhKRP-AS and Ehgfp were grown in the presence of $50 \mu \mathrm{g} / \mathrm{ml}$ of G418 for $48 \mathrm{~h}$. The amoebae $\left(10^{5}\right)$ were plated over a confluent CV-1 monolayer in 24 well plates for $1 \mathrm{~h}$. The adhering amoebae were removed by chilling and the remaining monolayer was stained with methylene blue and absorbance was measured at $660 \mathrm{~nm}$. The data is represented as percent (\%) destruction of the CV1 monolayer in comparison to untransfected HM-1 cells.

\section{ACKNOWLEDGMENTS}

Alka Mehra would like to thank CSIR for a scholarship. The authors would like to thank Dr. Rakesh Tyagi (Special Centre for Molecular Medicine, Jawaharlal Nehru University, New Delhi, India) for providing mammalian CV 1 cells. Research support from DST, DBT and CSIR, Government of India is gratefully acknowledged.

\section{REFERENCES}

1. Araiza-Orozco, L.M., E. E. Avila-Muro, M.L. Munoz, and S. Arias-Negrete, 1997. Entamoeba histolytica: role of surface proteases on its virulence. Arch Med Res. 28, 175-7

2. Mirelman, D., S. Moody-Haupt, S. Becker, Y. Nuchamowitz, R. Bracha, and R. Alon, R., 1996. Cell surface molecules of pathogenic and nonpathogenic Entamoeba histolytica and their relation to virulence. Adv Exp Med Biol. 408, 2257

3. Willhoeft, U., H. Buss and E. Tannich, 1999. DNA sequences corresponding to the ariel gene family of Entamoeba histolytica are not present in E. dispar. Parasitol Res. 85, 787-9

4. Espinosa-Cantellano, M. and A. Martinez-Palomo, 1991. The plasma membrane of Entamoeba histolytica: structure and dynamics. Biol. Cell. 72, 189-200

5. Petri, W.A. Jr., T. F. Jackson, V. Gathiram, K. Kress, L.D. Saffer, T.L. Snodgrass, M.D. Chapman, Z. Keren and D. Mirelman, 1990. Pathogenic and nonpathogenic strains of Entamoeba histolytica can be differentiated by monoclonal antibodies to the galactose-specific adherence lectin. Infect and Immun. 58, 1802-6

6. Simonishvili, S., S. Tsanava, K. Sanadze, R. Chlikadze, A. Miskalishvili, N. Lomkatsi, P. Imnadze, W.A. Petri Jr. and N. Trapaidze, 2005. Entamoeba histolytica: the serine-rich gene polymorphism-based genetic variability of clinical isolates from Georgia. Exp Parasitol. 110, 313-7 
7. Zhang, T. and S.L. Stanley Jr., 1999. DNA vaccination with the serine rich Entamoeba histolytica protein (SREHP) prevents amebic liver abscess in rodent models of disease. Vaccine. 18, 868-74

8. Kohler ,S. and E. Tannich, 1993. A family of transcripts (K2) of Entamoeba histolytica contains polymorphic repetitive regions with highly conserved elements. Mol Biochem Parasitol. 59, 49-58

9. Li,E., C. Kunz-Jenkins and S. L. Stanley Jr., 1992. Isolation and characterization of genomic clones encoding a serine-rich Entamoeba histolytica protein Mol. Biochem. Parasitol. 50, 355-357

10. Mai, Z. and J. Samuelson, 1998. A new gene family (ariel) encodes asparagine-rich Entamoeba histolytica antigens, which resemble the amebic vaccine candidate serine-rich $\mathrm{E}$. histolytica protein. Infect and Immun. 66, 353-5

11. Stanley, S.L. Jr., A. Becker, C. Kunz-Jenkins, L. Foster and E. Li, 1990. Cloning and expression of a membrane antigen of Entamoeba histolytica possessing multiple tandem repeats. Proc. Natl. Acad. Sci. U S A. 87, 4976-80

12. Haghighi, A., S. Kobayashi, T. Takeuchi, G. Masuda and T. Nozaki, 2002. Remarkable genetic polymorphism among Entamoeba histolytica isolates from a limited geographic area. J Clin Microbiol. 40, 4081-90

13. Hamann, L., R. Nickel and E. Tannich, 1995. Transfection and continuous expression of heterologous genes in the protozoan parasite Entamoeba histolytica. Proc Natl Acad Sci U S A. 92, 8975-9
14. Jacobs, T. and M. Leippe, 1995. Purification and molecular cloning of a major antibacterial protein of the protozoan parasite Entamoeba histolytica with lysozyme-like properties. Eur J Biochem.. 231, 831-838

15. Nickel, R., Jacobs, T., Leippe, M., 1998. Molecular characterization of an exceptionally acidic lysozyme-like protein from the protozoon Entamoeba histolytica. FEBS Lett. 437, 153-7

16. Fouche, P.B. and J.H. Hash, 1978. The N,Odiacetylmuramidase of Chalaropsis species. Identificaiton of aspartyl and glutamyl residues in the active site. J Biol Chem. 23, 6787-93

17. Aley, S.B., Z. A. Cohn and W. A. Scott, 1984. Endocytosis in Entamoeba histolytica Evidence for a unique non-acidified compartment. J. Exp. Med. 160, 724-737.

18. Alon, R.N., R. Bracha and D. Mirelman, 1997. Inhibition of expression of the lysine-rich $30 \mathrm{kDa}$ surface antigen of Entamoeba dispar by the transcription of its antisense RNA. Mol. Biochem. Parasitol. 90, 193-201

19. Orozco, E., G. Guarneros, A. Martinez-Palomo and T. Sanchez., 1983. Entamoeba histolytica. Phagocytosis as a virulence factor. J. Exp. Med. $158,1511-1521$

20. Bruchhaus, I., T. Roeder, H. Lotter, M. Schwerdtfeger and E. Tannich, 2002. Differential gene expression in Entamoeba histolytica isolated from amoebic liver abscess. Mol. Microbiol.44, 1063-72. 